\title{
Als Hebamme freiberuflich arbeiten
}

\author{
Anja Stamm, Heidi Kuntz
}

\author{
Die überwiegende Mehrheit der rund 24000 Hebammen in Deutschland arbeitet ganz oder \\ teilweise freiberuflich. Der Artikel beleuchtet, worauf es beim Start in die Selbstständigkeit \\ ankommt. Eine freiberufliche Hebamme vermittelt, welche Herausforderungen und Chan- \\ cen mit der Selbstständigkeit verbunden sind.
}

Der Deutsche Hebammenverband fasst in seinem Zahlenspiegel zur Situation der Hebammen 4/2019 zusammen: „Der DHV hat 19.862 Mitglieder, davon 16.468 aktiv (Stand: Februar 2019). Mindestens 13.388 Hebammen, die Mitglied im DHV sind, arbeiten (auch) freiberuflich als Beleghebamme oder betreuen außerklinische Geburten, arbeiten in der Wochenbettbetreuung und Vorsorge. Sie arbeiten zum Teil in Teilzeit und parallel zur Angestelltentätigkeit. Davon arbeiten 2.705 freiberuflich in der Geburtshilfe, das sind 20,2 Prozent." [1]

Zunehmend scheint die Freiberuflichkeit eine attraktive Alternative zur Festanstellung in einer Klinik, weiß Hadi AlWakil, Geschäftsführer der AZH Abrechnungszentrale für Hebammen $\mathrm{GmbH}$. Er berät mit seinem Team Hebammen beim Start in die Soloselbstständigkeit ebenso wie beim Wechsel aus dem Angestellten- ins Dienst-Beleghebammen-System. Bei diesem Modell wird die Kreißsaal-Organisation komplett von freiberuflichen Hebammen übernommen. Die Kolleginnen arbeiten wie vorher in einem gemeinsamen Dienstmodell. Der überwiegende Teil der kooperierenden Hebammen nutzt ein sog. Pool-System. Hierbei werden die abgerechneten Leistungen in einem gemeinsamen Einnahmentopf gesammelt und anteilig nach den eingebrachten Anwesenheitszeiten aufgeteilt. Das garantiert eine gerechte Verteilung und reduziert das Verdienstrisiko der einzelnen Hebamme.

„Wir informieren und unterstützen jedes Jahr zahlreiche angestellte Hebammenteams, die sich für das Belegsystem interessieren“, berichtet Al-Wakil. „Entscheidet sich das Team nach Aufklärung über die Vor- und Nachteile für weitere Schritte, begleiten wir die Verhandlungen mit der Klinikleitung. Außerdem unterstützen wir bei den internen Vorbereitungen für eine gut funktionierende Ablauforganisation.“ 2020 hat die Abrechnungszentrale für Hebammen $\mathrm{GmbH}$ Hebammen-Teams im Vinzenz-Palotti-Hospital Bensberg, im Klinikum
Bamberg, in der Fürst Stirum Klinik Bruchsal, im Universitätsklinikum Augsburg, im Klinikum Coburg und im Helios Klinikum Erfurt auf dem Weg ins Belegsystem begleitet. „Alle Beteiligten sind mit den Ergebnissen dieser Lösung sehr zufrieden“, so Al-Wakil.

Im außerklinischen Bereich berät das AZH-Team Praxen und Geburtshäuser, führt Tagesveranstaltungen an Hebammenschulen und in Studiengängen durch. „Aufgrund vermehrter Nachfrage vereinbaren wir mittlerweile auch Webinare mit Hebammen, die sich außerhalb oder nach ihrer eigentlichen Hebammenausbildung über den Weg in die Freiberuflichkeit informieren möchten.“

\section{Inhalte des Businessplans}

Wer die sichere Festanstellung für eine freiberufliche Tätigkeit als Hebamme hinter sich lässt, sollte vorab einige grundsätzliche Fragen abklären. Das gilt erst recht, wenn sich mehrere Hebammen zusammenschließen, deren persönliche Situation ganz unterschiedlich sein kann, z. B. was Familienplanung, finanzielle Rücklagen und Stand innerhalb der beruflichen Laufbahn angeht. Ein Businessplan hilft dabei, die Geschäftsgründung systematisch anzugehen. Er ist zudem i.d.R. die Voraussetzung, um Kredite oder Förderungen zu beantragen bzw. mit Kooperationspartnern zu verhandeln. Der Businessplan enthält die wichtigsten Eckdaten des Geschäftskonzeptes und der Finanzplanung, z.B.:

\section{- Geschäftsidee}

Welche Leistungen sollen angeboten werden?

- Gründerin(nen)

Wer wird freiberuflich als Hebamme bzw. Hebammen-Team tätig? Was ist die Motivation, sich selbstständig zu machen? Welche fachliche und unternehmerische Qualifikation, wie viel Berufserfahrung liegt vor?

\section{- Zielgruppe}

An wen richtet sich das Angebot (nur Vor- und 
Nachsorge oder auch Geburtsbetreuung, klinische oder außerklinische Geburtsbetreuung)? Welche Bedürfnisse haben die zu betreuenden Frauen und Familien? Wie groß ist der geplante Aktionsradius?

- Marktsituation

Wie hoch ist der Bedarf für eine freiberufliche Hebamme (bzw. das Team) in der Region? Wie entwickelt sich die Versorgungssituation perspektivisch? Welche Marktlücken bzw. Kooperationsmöglichkeiten gibt es?

\section{- Wettbewerbssituation}

Wie viele Hebammen(-Teams) gibt es im Umkreis? Worin unterscheidet sich ihr Angebot?

- Öffentlichkeitsarbeit und Marketing Wie soll das Angebot bekannt gemacht werden (z. B. Praxisschild, Kontaktaufnahme zu Gynäkologinnen und Kliniken, eigene Website, Social Media, Flyer, lokale Presse, Anzeigen)? Welche Kontakte sind zu knüpfen bzw. auszubauen?

- Standort

Wo werden die Leistungen angeboten (z. B. eigene Praxis, Praxisgemeinschaft, nur Hausbesuche, Beleghebamme in einer Klinik)? Wie sieht die Einrichtung der Praxis aus? Wie ist die Erreichbarkeit (Geschäftszeiten, öffentliche Verkehrsmittel, Parkplätze)?

\section{- Organisation und Ausstattung}

Welche Arbeitsschritte erfordern die einzelnen Leistungen und wie hoch ist der Zeit- bzw. Personalbedarf? Wie werden die Aufgaben im Team verteilt? Welche Regelungen sind ggf. mit der Klinik im Belegvertrag zu treffen? Welche organisatorischen und vertraglichen Vereinbarungen sollte das Team festlegen? Wie viel Zeit wird für fachfremde Arbeiten aufgewandt (z. B. EDV-Einrichtung, Bestellungen etc.)? Für welche Aufgaben wird externe Hilfe benötigt (z. B. Abrechnung, Steuerberatung, PC-Support etc.) Welches Praxis-Mobiliar und welche Betriebsmittel sind erforderlich? Was muss alles angemeldet werden?

- Rechtsform Wird die Hebamme als Einzelunternehmerin tätig oder gründet ein Team z.B. eine Partnerschaftsgesellschaft, eine Gesellschaft bürgerlichen Rechts (GbR), eine Gesellschaft mit beschränkter Haftung $(\mathrm{GmbH})$ oder einen eingetragenen Verein (e. V. )?

- Chancen und Risiken

Wie wird sich das Gründungsvorhaben mittel- bis langfristig entwickeln? Wie werden Risiken abgesichert (Haftpflicht, Rechtsschutz, Arbeitsunfähigkeit)?

\section{- Wichtige Planzahlen}

Welche Verdienstaussichten stehen dem Risiko der Freiberuflichkeit gegenüber? Wie hoch sind die Investitionen und laufenden Kosten (Hebammen-Ausstattung, Praxis-Mobiliar, Miete u. Nebenkosten, Telefon, Büroartikel, Kfz-Kosten, Fortbildungskosten, Kosten für Abrechnungszentrale bzw. Steuerberater, Haftpflichtversicherung, Beitrag Berufsgenossenschaft, Beitrag Berufsverband)? Wie hoch die eigenen
Lebenshaltungskosten? Wie hoch muss der Nettoumsatz sein? Wann ist die Gewinnschwelle erreicht? Wie werden Liquiditätsengpässe abgesichert?

\section{- Finanzplan}

Bei der Erstellung eines Finanz- und Kapitalbedarfsplanes für die ersten drei Jahre unterstützen z. B. Steuerberater und Abrechnungsunternehmen.

\section{CHECKLISTE}

\section{Vorbereitung zur Freiberuflichkeit}

- Anmeldung beim örtlichen Gesundheitsamt

- Abschluss einer Berufs-Haftpflicht-Versicherung

- Beantragung eines Institutionskennzeichens (IK)

- Beitritt zum Vertrag über die Versorgung mit Hebammenhilfe sowie Eintrag in die Vertragspartnerliste der GKV

- Anmeldung der freiberuflichen Tätigkeit beim Finanzamt

- Beitritt zur Berufsgenossenschaft (BGW)

- An- oder Ummeldung bei der Deutschen Rentenversicherung

- Abklärung Status Kranken- und Pflegeversicherung

Quelle: AZH Abrechnungszentrale für Hebammen $\mathrm{GmbH}$

\section{Pflichten freiberuflicher Hebammen}

Selbstständige Hebammen gehen nach §18 Einkommensteuergesetz einer freiberuflichen Tätigkeit nach, d. h. sie sind nicht verpflichtet, ein Gewerbe anzumelden. Allerdings müssen sie die Aufnahme ihrer freiberuflichen Tätigkeit dem Finanzamt und dem Gesundheitsamt gegenüber anzeigen. Berufseinsteiger müssen dabei die Berechtigung zum Führen der Berufsbezeichnung nachweisen. Das Gesundheitsamt ist zudem die vorgesetzte Dienststelle, d.h. die Amtsärztin bzw. der Amtsarzt ist der freiberuflichen Hebamme gegenüber weisungsbefugt. Hebammen in der außerklinischen Geburtshilfe sind verpflichtet, ein persönliches Hebammentagebuch zu führen.

Die Hebammenberufsordnungen der Bundesländer regeln weitere besondere Pflichten von Hebammen bei freiberuflicher Tätigkeit. So sind diese u.a. verpflichtet, eine Haftpflichtversicherung abzuschließen, die sie gegenüber Haftpflichtansprüchen im Rahmen ihrer beruflichen Tätigkeit absichert. Freiberufliche Hebammen müssen sich ferner auch an Maßnahmen der externen Qualitätssicherung für außerklinische Geburtshilfe beteiligen, z.B. an bundesoder landesweiten Perinatalerhebungen.

Seit Inkrafttreten des neuen Vertrags über die Versorgung mit Hebammenhilfe nach $\S 134 a$ SGB V sind freiberufliche Hebammen zudem verpflichtet, ein 
Qualitätsmanagement-System einzuführen [2]. Einen Übersichtsartikel dazu finden Sie in DIE HEBAMME 3/ 2018 [3].

Gemäß § 2 SGB VII besteht eine Pflichtmitgliedschaft in der gesetzlichen Unfallversicherung. Spätestens eine Woche nach Aufnahme der freiberuflichen Tätigkeit muss die Anmeldung bei der zuständigen Berufsgenossenschaft für Gesundheitsdienst und Wohlfahrtspflege (www.bgw-online.de) erfolgen. Im Versicherungsfall zahlt die BGW Verletztengeld bzw. (Teil-)Rente und Unterstützungsleistungen bei beruflicher Wiedereingliederung (Fortbildung, Umschulung, Kraftfahrzeughilfe, Wohnungshilfe, Reha).

Freiberufliche Hebammen tragen die Beiträge zur Kranken- und Pflegeversicherung allein. Im Bereich der Krankenversicherung besteht Wahlfreiheit zwischen der gesetzlichen oder privaten Krankenversicherung. Die Beiträge der gesetzlichen Krankenversicherungen sind abhängig vom Einkommen und den jeweils gültigen Beitragssätzen bzw. dem individuellen Zusatzbeitrag. Die Krankenkassen beraten auch über mögliche Wahltarife und Zusatzversicherungen.

Freiberufliche Hebammen sind gemäß §2 SGB VI verpflichtet, eine gesetzliche Alterssicherung abzuschließen. Zuständig ist die Deutsche Rentenversicherung (www.deutsche-rentenversicherung.de). Die Anmeldung muss spätestens drei Monate nach Beginn der Tätigkeit erfolgen. Die Beiträge errechnen sich aus dem steuerpflichtigen Bruttoeinkommen, im ersten Jahr auf Basis des geschätzten voraussichtlichen Arbeitseinkommens.

\section{Abrechnung von Hebammenleistungen}

Hebammenleistungen werden als Kassenleistung mit der gesetzlichen (GKV) oder privaten Krankenversicherung (PKV) direkt abgerechnet. Die Details fasst Hadi Al-Wakil nachfolgend zusammen:

Bei der GKV orientieren sich die abrechnungsfähigen Leistungen am Vertrag über die Versorgung mit Hebammenhilfe nach § 134a SGB V [2]. Voraussetzung für die Abrechnung ist, dass die Hebamme gemäß § 293 SGB V über ein persönliches Institutionskennzeichen (IK) verfügt, das sie bei der Abrechnung ihrer persönlichen Leistungen mit den Krankenkassen verwendet. Hebammen, die gemeinsam abrechnen, müssen dafür gesonderte IK führen. Die Hebamme muss das IK bei der Sammel- und Verteilungsstelle IK beantragen. Das Antragsformular ist als Download unter www.arge-ik.de verfügbar.

Voraussetzung für die Abrechnung mit der GKV ist der Beitritt zum Vertrag über die Versorgung mit Hebammenhilfe nach § 134a SGB V. Er erfolgt beim GKV über
Anlage 4.1. des Vertrags [2]. Freiberufliche Hebammen, die Mitglied im Berufsverband DHV oder BfHD sind, treten dem Vertrag über ihre Verbandsmitgliedschaft automatisch bei. Hierfür müssen sie ihre Berufserlaubnis, den Abschluss einer Berufshaftpflichtversicherung und die Anmeldung beim Gesundheitsamt nachweisen. Zudem muss die Hebamme sich in der Vertragspartnerliste der GKV eintragen (Anlage 4.2. [2]). Hierfür muss sie neben ihrem IK und ihren Kontaktdaten auch ihre geplanten Tätigkeitsbereiche (z.B. Schwangerenbetreuung, Wochenbettbetreuung, Kurse) angeben. Die gesetzlichen Kostenträger vergüten ausschließlich Leistungspositionen aus Tätigkeitsbereichen, die zuvor angemeldet wurden. Es ist sehr wichtig, alle relevanten Bereiche anzugeben. Im Rahmen der Abrechnung, die üblicherweise elektronisch erfolgt, reicht die Hebamme als Nachweis für erbrachte Leistungen neben der Rechnung Versichertenbestätigungen beim Kostenträger ein. Auf diesen bestätigt die Versicherte mit ihrer Unterschrift den Erhalt der abgerechneten Leistungen.

Die Abrechnung mit privat krankenversicherten Klientinnen funktioniert nahezu identisch. Mit Ausnahme von Sachsen hat jedes Bundesland hierfür eine Privatgebührenordnung formuliert. Die Gebührenordnungen lehnen sich inhaltlich an den Vertrag der GKV-Versicherten an. Hierbei können unterschiedliche Sätze in Rechnung gestellt werden (maximal 2,0). Die Anforderungen an die Abrechnung mit Selbstzahlerinnen stellen sich bezogen auf die notwendigen Anmeldevoraussetzungen weniger aufwendig dar. Hier reicht theoretisch die gestellte Rechnung über die erbrachten Leistungen aus.

Der Vertrag nach § 134a SGB V inklusive o.g. Anlagen ist auf folgenden Websites zu finden:

- www.gkv-spitzenverband.de [2]

- www.hebammenverband.de

- www.bfhd.de

BEST PRACTICE

Eine Hebamme fest anstellen

Heidi Kuntz ist seit 20 Jahren Hebamme mit eigener Praxis in Zweibrücken. Vor 5 Jahren stellte sie eine junge Kollegin direkt nach der Berufsausbildung ein. Worauf es dabei ankommt, beschreibt sie Punkt für Punkt:

Das Modell: Festanstellung einer Hebamme. Der Arbeitsvertrag wurde mithilfe eines Steuerberaters entwickelt und mit der Hebamme ausgehandelt. Arbeitszeiten und Gehalt: Die Angestellte arbeitet 8 Stunden/Tag, jedes zweite Wochenende hat sie frei bzw. Rufdienst für 8 Stunden/Tag. Ein realisierbares und faires Bruttogehalt wurde mithilfe des Steuerberaters errechnet. Er hat verglichen, was angestellte Krankenhaus-Hebammen verdienen, welche Einnahmen die Angestellte für die Praxis 
einbringen könnte und welche Ausgaben für sie anfallen (Sozialversicherungen, Haftpflicht, Leasing eines Praxisautos, Benzin, Kfz-Versicherung ...) Größte Herausforderung: Wenn die Mitarbeiterin krank ist oder in Mutterschutz geht, muss die Inhaberin ihr Gehalt eine zeitlang fortzahlen, obwohl die Einnahmen durch ihre Betreuung wegfallen. Aufgrund der 1:1-Betreuung kann die Inhaberin nicht mehr Geburten gleichzeitig betreuen. Deshalb sollte sie einen Puffer für Notfälle von 10.000 bis 15.000 Euro anlegen und sich mit weiteren Einnahmequellen entsprechend absichern. Heidi Kuntz bietet z.B. Kurse für Kinder und Schwangere an, die nicht von den Kassen übernommen werden, wie Babyschwimmen, Kinderschwimmen oder Personal-Training mit der Powerplate.

Vertretung im Krankheitsfall: Heidi Kuntz hat für ihre Praxis einen Belegvertrag mit der Universitätsklinik Homburg Saar abgeschlossen. Im Krankheitsfall übernehmen die Klinikhebammen die von ihr betreuten Frauen.

Vorteil für die angestellte Hebamme: Sicherer Arbeitsplatz, festes Einkommen, Eins-zu-eins-Einarbeitung, Absicherung bei Krankheit und

Schwangerschaft.

Welche Skills muss sie Hebamme mitbringen, um ein Unternehmen aufzubauen? Heidi Kuntz: Visionen, Ziele, sehr viel Mut und Women-Power. Außerdem sollte man immer einen Plan-B haben, wenn es mit Plan-A nicht läuft.

Wie geht's weiter: Heidi Kuntz expandiert weiter und will im Sommer ein Geburtshaus eröffnen.

Kontakt: www.familyinform.de
Autorinnen / Autoren

Anja Stamm ist freie Journalistin mit dem Schwerpunkt Gesundheitskommunikation in Frankfurt am Main.

Heidi Kuntz ist Hebamme, B.A. Gesundheitspädagogik, in 66482 Zweibrücken.

\section{Korrespondenzadresse}

a.stamm@redaktionspool.de

\section{Literatur}

[1] DHV. Zahlenspiegel zur Situation der Hebammen 4 / 2019. Im Internet: https://hebammenkongress.de/wp-content/uplo ads/2019/05/2019_04-Zahlenspiegel-zur-Situation-der-He bammen.pdf; Aufruf: 30.03.2021

[2] GKV Spitzenverband. Vertrag über die Versorgung mit Hebammenhilfe nach § 134a SGB V. Im Internet: https://www.g kv-spitzenverband.de/krankenversicherung/ambulante_leis tungen/hebammen_geburtshaeuser/hebammenhilfevertrag/ hebammenhilfevertrag.jsp

[3] Stamm A. Qualitätsmanagement für Hebammen. Die Hebamme 2018; 31(3): 140-146

Bibliografie

Die Hebamme 2021; 34: 40-43

DOI 10.1055/a-1400-3488

ISSN 0932-8122

(C) 2021. Thieme. All rights reserved.

Georg Thieme Verlag KG, Rüdigerstraße 14,

70469 Stuttgart, Germany 